\title{
Evolving and Augmenting Worth Mapping for Family Archives
}

\author{
Gilbert Cockton \\ School of Design \\ Northumbria University \\ City Campus East \\ Newcastle upon Tyne, NE1 8ST \\ Gilbert_Cockton@ hotmail.com
}

\author{
Dave Kirk \\ Department of Computer Science, \\ University of Nottingham \\ Jubilee Campus, Wollaton Road \\ Nottingham, NG8 1BB \\ dsk@cs.nott.ac.uk
}

\author{
Abigail Sellen and Richard Banks \\ Microsoft Research \\ Roger Needham Building \\ $7 \mathrm{~J} \mathrm{~J}$ Thomson Ave \\ Cambridge CB3 OFB
}

\{asellen,rbanks\}@microsoft.com

\begin{abstract}
We describe the process of developing worth maps from field research and initial design sketches for a digital Family Archive, which resulted in a more simple and flexible worth map format. Worth maps support designing as connecting by forming explicit associations between designs and human values. Two supporting worth-centred design resources were developed: one to organize field materials (a worth board) and another to simplify worth map structure (user experience frames). During this process, we identified and refined a range of design elements and relevant human values for initial conceptual exploration of an innovative table top computer application. We end with an evaluation of the process and outcomes, complemented with insights from subsequent applications of worth maps that support recommendations on worth mapping practices. The resulting worth maps and associated resources were (and still remain) valuable, but experiences during this and other uses indicate that further improvements are needed.
\end{abstract}

\section{Categories and Subject Descriptors}

H.1.2 User/Machine Systems.

\section{General Terms}

Management, Design, Theory.

\section{Keywords}

Worth-Centred Development, Designing as Connecting, Worth Maps, Worth Sketches, Worth Boards, User Experience Frames.

\section{INTRODUCTION}

HCI research addresses human interactions with computers, their design, and sometimes both. A focus on use extends attention beyond artefacts, but user- and usage-centred design are a limited axiological step beyond an exclusive craft focus for interaction design. Axiology is the study of the truly good things in life, i.e., value, values and worth, which provides a broader basis for identifying interaction design opportunities, guiding HCI research, and selecting relevant and worthwhile evaluation criteria.

(C) The Author 2009.

Published by the British Computer Society
The mathematical origins of computing favour essentialist approaches, manifested in guidelines, patterns or heuristics that identify content, structure and properties of intrinsically good designs. Most $\mathrm{HCI}$ is antagonistic to such intrinsic value, instead locating value in human agency. Constructing design solely as the creation or conception of artefacts locates value intrinsically, i.e., in an artefact and not in the world of use and values. This conflates means and ends, using the same concepts to describe both, with the axiological consequence of severely restricting how human values are considered during design and evaluation.

Once we give equal attention to means and ends, we can separate design means from human values [9] (design ends [13]). Such values can be subsumed within a focus on worth, i.e., a positive balance of benefits over costs. An additional focus on worth can separate design purpose from the design itself, and can also enable evaluation criteria distinct from artefacts and usage. When we return attention to designed artefacts, as we must, we then have two foci, on design means and ends. This generates a space that moves design beyond a craft practice to one referred to as linking, connecting, translating or bridging [5]. Having separated design means and ends, we have to reconnect them, resulting in a shift from designing as crafting, to designing as connecting.

Interaction is largely a means to an end, and very rarely an end in itself. HCI has not sufficiently extended artefact-centred craft approaches with axiological ones centred on worth and human values. To do this, key questions must be answered, including:

- How do we settle on design purpose?

- How do we express design purpose in ways that are not intrinsic to the artefact or interaction?

- How do we link choices of means to choices of ends?

The starting point for exploring these and other questions was:

- The results of a field study on the treasured objects (including photographs) that households keep;

- Initial design sketches for a digital Family Archive that would enable new practices and experiences when preserving, enhancing and sharing valuable objects;

- A modified approach from consumer psychology (worth mapping) that relates chosen means to chosen ends.

The first two elements of this starting point are next briefly summarised. The third element is then introduced. Experiences and outcomes during the worth mapping process are then 


\section{G. Cockton et al.}

presented. Wins and issues are next summarised, before concluding with recommendations for worth mapping practices.

\section{A DIGITAL FAMILY ARCHIVE?}

Interesting diverse challenges arise from the opportunity to develop systems that allow input and safe archiving of both digital and physical artefacts, as well as natural interaction with those media. Families interact with, manage, and archive materials that are important in preserving and sharing memories. Such materials include photographs, videos, object collections, heirlooms, and mementoes such as children's art and letters. These are currently stored in different parts of homes with varying organisation, accessibility and protection. Objects are kept for different reasons and with different consequences. Our collaborative research explored whether worth maps could help us to form associations between design interventions and family archiving purposes. In addition, because worth maps were still essentially untried and evolving, a second (but not secondary) aim was to use family archiving as a new context to try out and refine worth mapping.

\subsection{Understanding Family Archiving}

Microsoft Research Cambridge's Family Archive research is grounded in existing in-depth studies of how families capture, edit, manage and archive both digital photos and videos [11,12]. In order to understand family archiving more broadly (including the physical objects that households cherish), we carried out a focused field study with a balanced range of 11 families: young couples with no children, families with children (including variously, infant, pre-school, elementary school and high school age groups), older couples whose children had left home, and one widower living part-time with adult children. In each family at least two people participated in discussions: one person who was usually determined to be the person most in charge of organizing the family's 'archive', and at least one other family member. Mostly, all adults and several children (where present) contributed to discussions. Active participants ranged in age from 5 to 70, across a variety of backgrounds and occupations.

Discussions fitted into visits where the second author explored people's homes, examining and discussing objects of sentimental importance to the family, both digital and physical in form. This was facilitated by a guided tour of the home that normally lasted between 60 and 90 minutes, in which participating family members showed objects in every room of their house (excluding inaccessible lofts or garages). Prior to the start of the tour, we discussed what we might mean by "sentimental items" (i.e., if it was special in some way, more than purely decorative or functional), but with ambiguities resolved during tours. In all cases, participants were keen to show their artefacts; even those boxed away or in other storage, and would often resort to pulling objects out of various semihidden places. Shown items were photographed. The researcher avoided questions about items, but typically participants spontaneously produced accounts of what the objects were, giving their associated history, and often explicitly stating why they were being kept there. Participants were also asked about the nature of things kept in more inaccessible places (such as lofts) to compare what was visible. At the most appropriate point in the tour (usually in the presence of a computer), participants were also asked for details of the kinds of digital artefacts that they kept for sentimental reasons. This usually included prompting participants to consider digital photos and videos, emails, and archived digital work. This would often lead to discussion of other digital devices that might store sentimental items, e.g., cell phones, answer machines. Again, photos were taken of relevant screen shots or devices.
Having determined what items were actively archived (and to a large extent having already been told why items were being kept), follow-up interviews were conducted to clarify why different items were kept. A second visit, again lasting between 60 and 90 minutes, was made $1-2$ weeks after the initial visit. Participants engaged in an informal semi-structured interview in which they were shown some photos from the guided tour (usually picked to represent different categories of objects emerging in the data). Participants were asked to give more detail about how they felt about those objects, and why and how they had kept them. Participants were also encouraged to think about the nature of differences between physical and digital artefacts, and were asked whether a digital copy of an item under discussion could substitute for the original physical item, or indeed if items could be enhanced in some way by association with other digital media. Questions about who had control of the family archive and who should have access to items within it were also broached to explore issues of how collected artefacts were merged and managed within the family. The collected data were then analysed as a source of human values for subsequent worth mapping.

\subsection{Envisaging a Digital Family Archive}

As the field data were being analysed, organised and interpreted, brainstorming sessions were facilitated by an interaction designer (fourth author), with input from human science researchers (second and third authors) and hardware and software engineers. A set of annotated sketches resulted, which provided design options for consideration for worth mapping. All options were initially focused on a multi-touch table top computer as a platform for a family archive. At this point, the facilitator (first author) began a three month research visit to explore how worth mapping could structure, extend and inform designing by translating, bridging and linking between craft means and human ends.

\section{WORTH MAPS}

The research collaboration provided an opportunity to use worth maps to support an ambitious design research programme involving a large multi-disciplinary team. Worth mapping could be informed by co-ordinated design and field studies. Up to this point, published worth maps had been speculative, hypothetical or autobiographical. They had not been used in a live design setting, but had nevertheless been progressively adapted from their 'seed' form of Hierarchical Value Models (HVMs) [1]. This section briefly outlines this evolution, and introduces a range of terminology that will be new to most HCI readers. A more detailed account of the initial adaptations to HVMs is given in [4].

HVMs use Means-End Chains (MECs) to associate product attributes with personal values. HVMs use laddering techniques from psychology to explore consumer associations between means and ends. Starting with a focus on product attributes, laddering interviews next elicit consequences of product ownership and use, and then the personal values that such consequences support. A MEC thus results, which begins with product attributes as means, continues with ownership and usage consequences as further means, and ends with personal values. An HVM is a diagrammatic integration of separately elicited MECs. This is based on merging MECs at shared consequences or personal values, resulting in a hierarchy with personal values at the top and product attributes at the bottom. If negative MECs are elicited, a second hierarchy will converge downwards to negative values (or aversions). 


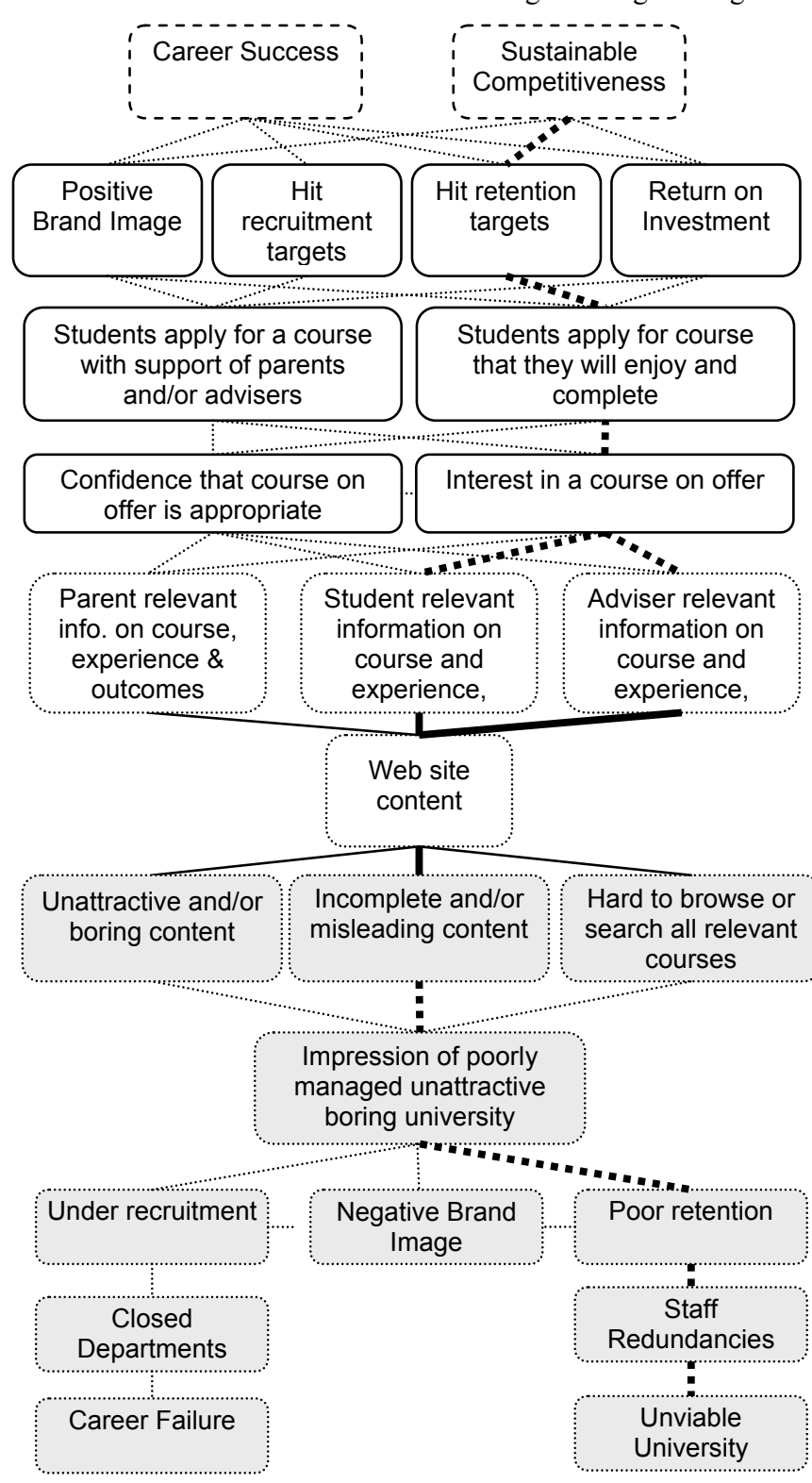

Figure 1: Example VC's W/AM for a University Web Site [4]

A new representation, Worth/Aversion Maps (W/AMs [4]) took advantage of two decades of experience with HVMs. This revised the underlying structure of HVM MECs, which began as a fixed structure of product attributes, consequences and values. The means of product attributes result in consequences from ownership and usage, which in turn satisfy values, which are the ends to which product attributes and consequences are means. There are two types of means-end chain element (MECE) for each of these categories (product attributes, consequences, values). Product attributes can be concrete or abstract. W/AMs retained both these MECE types. Consequences can be functional or psychosocial. W/AMs refined/extended these with further types (psychological, physiological, social, financial, environmental). HVM values were restricted to 18 instrumental and 18 terminal values from Rokeach's Value Survey [14]. This primes respondents during laddering, eliciting human values that would not otherwise be articulated. W/AMs thus imposed no vocabulary on the worthwhile outcomes that formed the ends of their MECs. Also, the rigid ordering of functional to psychosocial consequences imposed a primacy of action over meaning, when action can equally be a consequence of meaning. Basic HVMs were thus overly constrained, potentially distorting MECs that they integrate.
Figure 1 shows a hypothetical W/AM for a Vice-Chancellor (VC) in relation to a university web site. It is a 'destinies' W/AM, that emphasises potentially relevant outcomes and usage consequences, relating these to a minimal set of product attributes. Concrete product attributes are thus reduced to 'web site content'. Six abstract product attributes (three positive above 'web site content', three negative below) indicate that web content should be relevant, attractive, complete, and must not be incomplete, boring or hard to browse/search. Negative abstract product attributes will have poor consequences on impressions, brand image, recruitment and retention, resulting in adverse outcomes of department closures, staff redundancies, an unviable university and $\mathrm{VC}$ career failure. Conversely, positive abstract product attributes will have good consequences for confidence, interest, student applications, brand image, recruitment and retention targets and return on investment, resulting in worthwhile outcomes of sustainable competitiveness and VC career success.

In the month before the research collaboration, W/AM usage was explored in a workshop for the Finnish national VALU project on user values in design [8]. Four groups identified MECEs from four HCI research papers. It became clear that distinctions were needed between form factors and software features. As a result, the last remaining MECE types from classic HVMs (concrete and abstract product attributes), were refined into two types of concrete attribute, materials and features, with, positive and negative abstract product attributes re-named to qualities and defects respectively. All HVM MECE types had thus been restructured and renamed. The combined effort of participants in the Finnish workshop was around 20 person hours, making this a valuable exercise in terms of formative evaluation.

Table 1: Positive MECEs for Designing as Connecting

\begin{tabular}{|c|c|c|c|}
\hline Worth Maps & W/AMs & HVMs & Focus \\
\hline \multicolumn{2}{|c|}{ Worthwhile Outcome } & Terminal Value & \multirow{4}{*}{ Human } \\
\hline $\begin{array}{c}\text { User } \\
\text { Experience }\end{array}$ & \multirow{3}{*}{$\begin{array}{c}\text { Usage } \\
\text { Consequences }\end{array}$} & $\begin{array}{c}\text { Instrumental } \\
\text { Value }\end{array}$ & \\
\hline Feeling & & $\begin{array}{l}\text { Psychosocial } \\
\text { Consequence }\end{array}$ & \\
\hline Action & & $\begin{array}{c}\text { Functional } \\
\text { Consequence }\end{array}$ & \\
\hline Quality & \multicolumn{2}{|c|}{ Abstract Product Attribute } & \multirow{3}{*}{ Design } \\
\hline Feature & \multirow{2}{*}{\multicolumn{2}{|c|}{ Concrete Product Attribute }} & \\
\hline Material & & & \\
\hline
\end{tabular}

W/AM's extensive consequence MECE types were a distraction during the Finnish workshop too. An alternative structure was thus used for usage consequences, based on a simple structure for user experience (UX) as an interplay of feelings and actions. The revised form of W/AMs was called a worth map, to remove an oversight within the initial motivation for worth-centred development (WCD) [3], where worth was used as both a noun synonymous with value (positive outcomes) and as a predicative adjective expressing a relation: the benefits are worth the costs. The latter is the preferred sense of worth for WCD, and this subsumes aversions (as costs). The simpler name of worth maps removed confusions introduced in [3] and compounded in [4]. Table 1 shows the evolution of MECE types. Ends are distinguished by italics. Negative elements are not shown, i.e., defects (design means), adverse consequences (human means) 


\section{G. Cockton et al.}

and adverse outcomes (human ends). The rightmost column indicates which MECE types are for design and human elements.

The collaboration began with worth maps that used the MECE types in the leftmost column. Not surprisingly, people new to MECs can be confused by the vocabulary in Table 1 . While strategically MECs and associated models or maps do offer attractive support for designing as connecting, there is an implicit warning here that MECE type names are tricky, with uncertainties and ambiguities amplified in use by worth mapping novices.

\section{A WORTH MAPPING CASE STUDY}

The collaboration reported below was realised via seven two hour worth mapping sessions. In between each, the facilitator documented the outcomes of the previous sessions, tracked the current state of the worth map and/or its constituent elements, and revised supporting tutorial and background material. An initial sequence of sessions isolated MECEs. The main sessions focused on worth mapping. A final session reviewed the worth mapping experience, presenting an innovative solution to problems that replaced all usage consequences with a single user experience MECE type. The total participant effort, excluding facilitation time, documentation and reflection, was around 85 hours for the MECE identification and worth mapping sessions, with an untracked amount of time in individual preparation and short informal follow up discussions.

Before attempting any worth mapping, existing field work and design sketches needed to be re-expressed as MECEs. In preparation, the mapping facilitator drew up rough lists of design elements (materials, features, qualities) from design sketches. Also, the draft of a paper on the field research was used as a starting point for rough lists of outcomes, actions, feelings, and experiences. Both sets of lists were informed by separate informal discussions with the field researcher, interaction designer, and software and hardware engineers. The aim was to produce initial MECEs for discussion and revision to feed into worth mapping.

At the first initial session with the project team, the interaction designer presented outputs from earlier design sketching sessions. Following this, the mapping facilitator (first author) motivated worth-centred approaches to design and evaluation (in terms similar to Section 1 above), and presented the initial rough lists of MECEs in a PowerPoint presentation, which were then discussed. The project lead (third author) asked if some definitions or questions could be prepared to help team members discriminate between different MECE types. A set of questions was later circulated, revised and then integrated into a short tutorial on worth mapping, MECs, worth-centredness and designing as connecting. This tutorial material formed the front matter of a 'living document' on evolving worth map elements, which was updated after each mapping session to present the current MECE set, keep track of issues, and record the change history. It was circulated before each session over the next six weeks.

For two further weekly exploratory sessions, MECE lists were prepared as a PowerPoint stack (one colour-coded MECE per slide, including some blanks), but rather than project and present, it was printed out as handouts (two slides/page) and cut up. In the first session, we walked through the living document and then laid out the set of 'cards' for each MECE type, forming a consensus through discussion, questions and clarifications. This preliminary to worth mapping was successful, in that we were able to add an overlooked positive outcome and six further valuable consequences. Also, two valuable consequences were 'promoted' to worthwhile outcomes. Such additions and promotions indicated that the questions developed to discriminate between different MECE type, when combined with facilitation, enabled a working grasp of the nature and role of each MECE type. The 'promotions' were of further importance in communicating the flexible boundaries between means and ends. Nothing is inherently a means or an end. What the mapping facilitator initially regarded as usage consequences were convincingly argued to be outcomes by those with direct experience of the field research and previous related studies. Ultimately, it comes down to the design context. Project teams must choose their design purpose, and thus privilege some aspects of design as ends, while demoting others to means.

In WCD, outcomes are bases for evaluation measures and targets $[4,6]$. It is thus worth [sic] exploring outcome subtypes to focus evaluations. Outcomes were distinguished as occurring in the home, through people, or in objects. Thus, after family archive usage, a home could become less cluttered, heirlooms could become better protected, or family members have better empathy for each other. The first two usage outcomes here could be directly observable through changes between home visits, whereas the third needs accounts of shared usage experiences. These distinctions actually arose from a common page format in the living document. A separate page recorded the current MECEs for each type. The three column format for UX MECEs (actions, feelings, experiences) was appropriated to structure other types (to be candid, it looked good). Outcomes and qualities too were split into three subtypes. Interestingly, this serendipitous use of a three column format exposed potential associations between MECE types. For example, qualities as anticipated interactions with the archive were strongly associated with initial UX feelings.

Structurally, the most important decision was to create feature subtypes. As element cards were laid out on the table, it soon became clear that we would have to modularise the planned worth maps. It had been a struggle to lay out the full MECE set on a large table in a meeting room with a capacity of around 25 used for research presentations. Space had to be created during the second half of the second initial session to make room for new cards, resulting in inelegant clumps of 'stuff we'd already looked at'. This could, and was, partially addressed for future sessions by halving cards in size (four/page), what was more important was exploration and agreement of a phase model for using a family archive, based on whether a treasured object was being captured, organised, edited or consumed. As there were four phases, not three, the living document's page format diversified slightly. The expectation was that by focusing on feature groups for each worth map, we could stay comfortably within the bounds of a large table. Feature subtypes were not subsets, as features could be common to more than one life cycle phase of an archived family treasure.

The second initial session thus had valuable outcomes in terms of the MECE population, consensus on their types, and agreement on subtypes invented to maintain a consistent page layout in the living document. The third and last initial session was a consolidation, motivated by absences at a conference for the second session. This let the project team walk through the MECEs one more time before moving to worth mapping. Receptiveness [6] to potential UXs was extended by considering existing home devices (e.g., the Whereabouts Clock [2]), adding three further valuable user experiences. We also brainstormed on opportunities enabled by wide area network links as a basic 'material' for a table top computer, and added further features that had not been identified in earlier design brainstorming and sketching sessions.

Thus the initial sessions could be regarded as a success. The most compelling evidence here is that we were able to continue 
to worth mapping, but the reasons for this lay in the valuable outcomes identified above, i.e., that MECEs could be:

1. derived from existing design sketches and user research, presented by a facilitator, and revised through collaboration.

2. extended by independent reflection and group discussion

3. re-typed through evidence-based discussion

4. re-structured, especially through novel subtypes that connected with wide background knowledge in the team (especially on usage phases $[11,12]$ )

The facilitator thus used three initial sessions to introduce MECEs to a team of two human scientists, an interaction designer and up to five technical specialists (software and hardware engineers). Useful insights emerged before focussing on worth mapping:

1. The spread of MECEs from materials to outcomes leveraged everyone's knowledge and expertise, e.g., materials and features that were not 'grounded' in existing field work could be added through thinking about use (e.g., lighting for a camera). Immediate association with human values was thus not the only ground for considering a design element. Design coherence and envisaged use could also provide initial rationales for roles in later MECs.

2. The materiality of the paper MECE 'cards' extended collaboration beyond talk. We could point at and move cards during discussions, and make notes on them.

3. The large table let most of us physically engage with MECEs. People could remain seated, stand up, or move around, providing cues about intent, focus, engagement and allegiance, letting the less active be re-engaged.

4. No table will ever be big enough, so you have to modularise worth maps. We subtyped features into four usage phases.

5. Tutorial material is very important, even with an approach's inventor present. This not only communicates key motives, knowledge, theories and concepts, but distinguishes situated contingent practices from 'method', discouraging the interpretation of evolving contextualised procedures as 'the worth mapping method'.

\subsection{Main Sessions and Supporting Work}

Three two hour sessions were held over the next month. At each, only features for one usage phase (i.e., capture, organise, edit or consume) were included as active MECEs. The facilitator laid MECEs out on the table, starting with a different type at each session each time (e.g., features, outcomes). The aim was to demonstrate worth mapping's flexibility, as some collaborators rightly had concerns about rigid prescriptive 'methods'.

In each session, worth sketches [5] were formed, i.e., worth maps without association arrows (invented opportunistically four months earlier for a rushed presentation where the first author ran out of time to include any arrows!) In worth sketches, MECEs are laid out in rows by type (e.g., qualities) and arranged vertically to suggest MECs [5]. Physical cards let us overlap adjacent MECEs, e.g., overlapping materials and features, features and qualities, qualities and feelings, feelings and experiences, and experiences and outcomes, and thus vertically expose MECs. At the end of each session, card overlaps were marked with irregular lassoed symbols to aid sketch reassembly. Photos of the worth sketch were also taken to aid reconstruction (Figure 2 below, from second main session). Initially, worth sketches were reassembled on the facilitator's glass corridor office wall. These were later transferred to large portable foam boards (approx $2.5 \times 1 \mathrm{~m}$ ) The worth sketches were then converted into partial worth maps using Microsoft Word's very primitive drawing tool, only adding associations discussed during a mapping session. Reassembling the sketch and creating a partial worth map diagram could take up to a day's effort, with a further half day for updating the 'living document'. The latter however, was a research tool to inform future development and use of worth sketches and maps, and would be omitted in faster paced design settings.

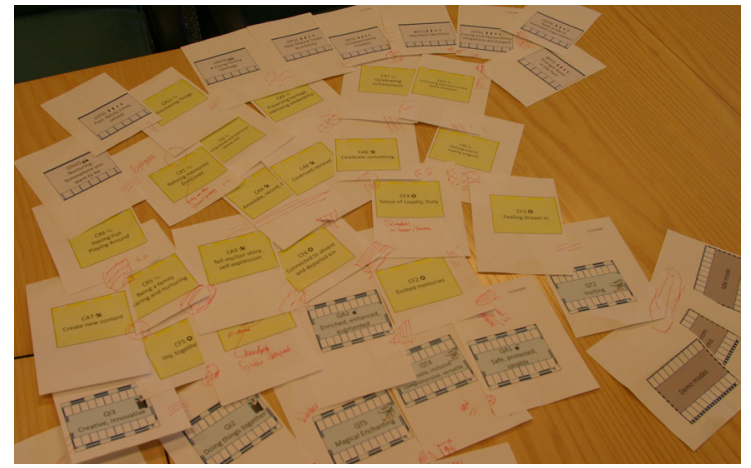

Figure 2: End of Session Photo of Worth Sketch

At the first main session, artefact capture features were associated in a worth sketch with materials and qualities. For example, during capture, artefacts would be automatically uploaded (e.g., from a mobile phone or digital camera) or require manual intervention (e.g., by placing a physical artefact on the tabletop). Some automatic tagging and grouping could happen at this point. Two design features from initial sketching were re-factored to simplify MECs. A key outcome of this session was a major reduction in potential qualities, achieved through grouping, including a sidelined group of commercially oriented 'brand' qualities outside of the scope of a research programme. Four groups of qualities remained in scope:

1. Ease of learning: self-explanatory, guiding, suggestive, familiar, intuitive, supportive

2. Relaxed use: accessible, at hand, suggesting casual, efficient, calm and easy capture

3. Magical play: playful, fun, magical, enchanting

4. Versatility: capable, comprehensive, inclusive

Valuable extensions and restructurings in initial sessions were thus further complemented by feature refinements (splitting), quality merging (coalescing) and deferral (brand qualities). Also, a worth sketch was successfully formed, enabling continuous team discussion on priorities, realities, possibilities and credibility.

They second main session, which focused on the organising phase (a.k.a. processing or management) of family archiving, when groupings and tags for uploaded artefacts could be edited, revised or extended, including audio annotation of photographs. Also, new links and associations could be created between archived items. This could support separate virtual archives for each family member, allowing artefact sharing without a single organising structure or common tag set. Several new materials were added as a result of discussions, both for audio annotations (e.g., a microphone) and for identifying different family members. Identifying the need for a microphone raised issues on sound management. MECE additions were generally placeholders to prompt later refinements, e.g., the table form was generalised, without commitment to anything specific (e.g., 


\section{G. Cockton et al.}

coffee table, butcher's block, hall table). Materials for the fourth consumption phase were also considered as a natural extension of organising (e.g., remote situated displays), and also materials related to generic stewardship concerns that pervaded object capture, management and editing (e.g., a drawer with integral hard disk for rapid removal during emergency). The importance of hardware materials dominated this session, complementing and extending prior design brainstorming and sketching. The main value of this session was identification of additional material MECEs, some through looking ahead to the consumption phase. No MECEs were discarded, coalesced, grouped or split in the second session. MECE population and structural stability at this point reduced the time needed for facilitator follow up, creating time to prepare a Worth Board to group evidence from field work to strengthen grounding of valuable outcomes in primary data.

Worth Boards extend mood boards for designing as connecting. While mood boards (as used in fashion, graphic and interior design etc.) collect options for features and materials to visually evoke desired qualities, worth boards focus on human outcomes and experiences. They are multimedia tools that gather together evidence for human elements, including photos, videos, audio recordings, interview text fragments and secondary research materials (e.g., trends data). Ideally, worth boards would be implemented on large interactive displays situated to maximise their support for design intelligence and discussion, as well as nurturing common ground across a project team. For speed, a Worth Board was implemented as a PowerPoint stack. The slide sequence was structured by valuable outcomes, with relevant photos and interview text fragments collected for each outcome. 118 slides of primary data were preceded by a short introductory orientation. Many slides contained one photo and caption, but others used photos and/or interview quotations to communicate grounded design goals for supporting family archiving.

Through engaging directly with primary data with the field researcher's support, the mapping facilitator revisited the three groups of outcome elements. Grounding in primary data reduced outcomes in each group, as indicated in brackets (before/after):

\section{Outcomes for families (10/9)}

2. Outcomes for homes $(7 / 4)$

3. Outcomes for treasured objects (6/4)

These groups act as a form of triangulation, representing similar human values from separate perspectives of people, places and things. For example, an outcome for families, Richer in spirit/cash just generalised other outcomes, and was thus dropped. The nine remaining valuable outcomes for families were:

\section{Manifest identities}

2. Increased family empathy

3. New shared times as a family

4. Manifest status for external social standing

5. Past family times relived

6. Caring and stewardship obligations discharged

7. Stronger roots in the past

8. Newly liberated from clutter and loose ends

9. New pride in improved home organisation
A further analysis distinguished between outcomes that were fairly well supported by existing household practices and artefacts, and those that would plausibly manifest or enhance 'new' value (indicated by words such as 'new(ly)', 'novel', 'better' or 'more') Three are italicised in list above. Three outcomes for houses were also seen as innovative, as were two for treasured objects. Such innovative outcomes can focus design research away from what is already well supported by existing practices (effectively 'reinventing the wheel'). The worth board was augmented with rationale slides for each claim of value innovation or extension.

At the third main session, the Worth Board was presented. Its format and content allowed relatively rapid presentation as it was close to a slide show in the $35 \mathrm{~mm}$ sense. The team then located artefact editing features into a third worth sketch of creative use, where copies of uploaded artefacts would be edited and combined to form new artefacts. This could involve (semi-) automatic photo collages, or repurposing for greetings cards, projection, situated displays, MMS messages to mobile phones, web-sites, multimedia presentations, or digital restorations. Features were added to support searching (e.g., tag clouds) and visual indications of the safety of original archived objects, with edits clearly always applied to copies. It was not clear whether these were 'new' features, or had just not made it into initial design sketches and follow on discussions. Either way, they were now added as MECEs and into the third worth sketch. It also became clear that some features from initial design sketches did not fit into the existing four usage phases, so a fifth entice phase was added to group features for a family archive that aimed to attract use, in ways similar to attract sequences of public information and service kiosks that entice passers-by to walk up and use.

The facilitator's expectation for the third main session was that it would be more efficient than the previous two, since the qualities population had been culled in the first session and the outcomes population through Worth Board construction. The MECE types that had replaced HVM and W/AM consequences (feelings, actions and experiences) however had constantly expanded, becoming unmanageable. As a result, shallow domes formed on the table as feelings, actions and experiences were interleaved into clusters (green cards, Figure 2). Fewer new design elements were added or re-factored relative to previous sessions, which in part was due to time spent on presenting the worth board. At this point, the research focus moved from the first aim (supporting design for family archives) to the second (getting worth mapping to work).

\subsection{The Consequences of HVM Consequences}

Difficulties in managing feelings, actions, and experiences MECEs forced reflection on worth maps roots in HVMs, which were initially targeted at advertising messages and market positioning in mature markets where marketing and communications were more influential than product or service innovations. Recent applications to digital services such as instant messaging [1] have retained the relatively passive reactive causal model of MECs underlying HVMs, with a hard determinism that product attributes will lead to usage consequences that will satisfy personal values. A simplicity of association that works for advertising and positioning works less well for proactive use of interactive digital artefacts, where a soft determinism [10] follows from human agency, which is as much a part of the 'materials' of interaction as multi-touch surfaces or digital cameras. Unmanageable complexity reflected the gap between hard and soft determinism. Simple MECs are suit the former, but not the latter. 


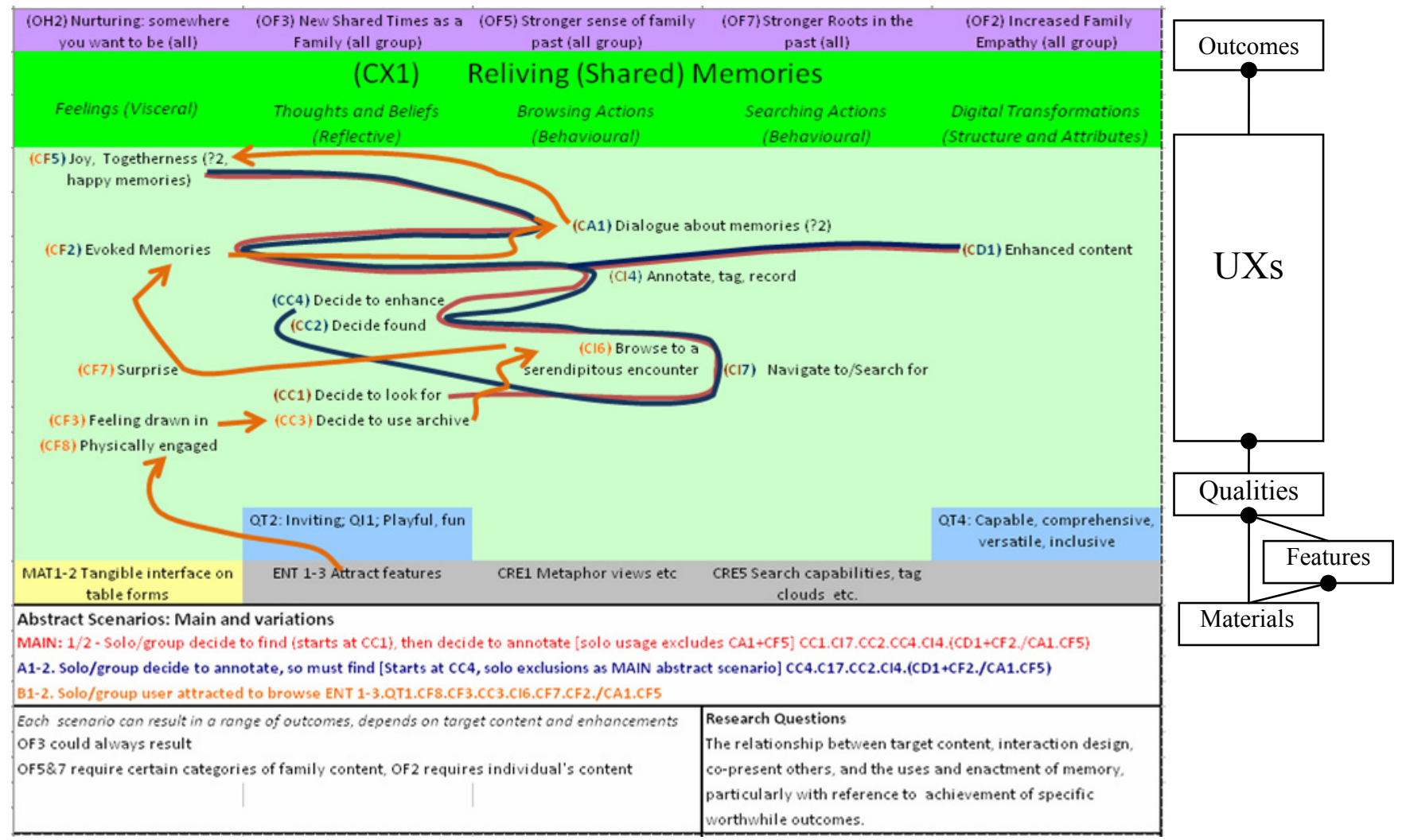

Figure 3: Editing Phase UEF for Reliving (Shared) Memories (left) with simplified MEC structure (right)

In a search for a more appropriate structure to replace HVM usage consequences, the facilitator experimented with a sub-map containing only feeling, action and experience MECEs. The credibility of actions and feelings was tested by associating user actions with features, and feelings with qualities, testing the fit of consequence to design elements. Next, a hierarchy was formed with UXs resulting from feelings and actions. These were laid out and fixed to a large foam board in a landscape orientation. However, to form adequate MECs, a fourth MECE type of beliefs was needed, e.g., a belief that a digital copy is "good enough", or "better for display" or lets the original be put somewhere safer, sold or otherwise disposed of. The latter action in a physical, social or economic context is quite distinct from interaction with a digital artefact. A UX segment of a MEC would thus need to be composed from feelings, usage actions, system responses, world actions and beliefs, and perhaps further categories [7]. Adding so many new MECE types to worth maps would make them impossible to manage (especially on a table, however big).

The response was to reduce usage consequences into a single UX MECE type, greatly simplifying worth maps and sketches. A separate novel representation was created to communicate the internal dynamics of a UX. The resulting User Experience Frames (UEFs, Figure 3) use a table format, with a flexible column structure spanning different categories of usage consequences. Column categories could be chosen to constitute a specific UX. For example, there are separate usage action columns for browsing and searching in Figure 3. Headers and footers span the columns, creating a 'window frame' structure. Headers grouped outcomes that can result for UXs. Footers group features and qualities that 'participate' in UXs. Also, in Figure 3, the right of the subfooter notes research questions for exploration in the context of the UEF. UEFs remove substantial complexity from worth maps and sketches (compare right of Figure 3 to Table 1, round line ends indicate 1-to-many associations. Figure 3 shows an editing phase UX for Family
Archive usage, when copies of stored artefacts can be annotated (e.g., tagging, text notes, voice recording) or creatively repurposed for future 'consumption.

Rough UEFs were prepared as spreadsheets, with 'scribbled' paths showing abstract scenarios, starting at the bottom, above relevant features and qualities, and threading through columns of feelings, beliefs, actions and responses, ending at the top with one or more valuable outcomes. Such outcomes are the happy endings of worth delivery scenarios [3,4,7]. Each abstract scenario provides a skeleton for several concrete scenarios. Several abstract scenarios can be threaded through one UEF, and can be named, specified as regular expressions, and associated with constraints specified in the footer. This enables a new range of analyses and critiques, and also provides 'sketching' support for a range of further design activities such as scenario authoring and experience prototyping.

\subsection{Final Session}

Before the final mapping session, there was a separate focused demonstration and discussion of the addition of 'physics' to the table top software [16], which would enable features that let objects be piled up, bounced or flipped. The current three partial worth maps were sufficiently well developed to enable discussion of how such features would contribute to the UXs that bridge between design elements and valuable outcomes. Prior group discussions during worth mapping provided much of the value here, rather than the maps themselves.

At the final session, the mapping facilitator motivated and presented UEFs, before laying out the worth map elements for the consumption phase of family archive use. In this phase, family members take advantage of previous archive management and creative/curatorial activities to share archive contents. A wide range of 'stuffcasting' opportunities were considered, such as uploading to the web (e.g., for sale, to a hobby or interest web-site, for materialisation on a mug, jigsaw, t-shirt etc.), distribution to situated displays (in the 
same/other homes), projection, printing, or MMS distribution to mobile phones. Most of these opportunities for materialisation had been identified during the hardware discussions for the second organising phase.

UEFs required reduction of the current MECE set and the three partial worth maps. There had not been enough time to do this before the final session, so after exploring UEF formats and discussing aspects of the consumption phase, for the rest of the session we discussed what had and had not worked during the exploratory worth sketching. The next section reports on themes arising from these discussions, feedback from a later retrospective presentation to the host research group, and the facilitator's personal evaluation. Where relevant, experiences with subsequent worth mapping independent of the facilitator are noted (e.g., [8]).

\section{WINS AND ISSUES}

The four main sessions provided a context for the first construction and use of a substantial well grounded worth board, and for invention of a new design representation, UEFs, which can be used independently of worth sketches and maps, either to add heart to task models, or to avoid writers' cramp in scenario authoring (see [7]). Although worth maps demonstrated strong potential, the collaboration also highlighted challenges that must be addressed. Positive potentials are considered first.

\subsection{Win 1: Connecting to Innovative Purpose}

The roots of the English word 'design' lie in the French word 'dessiner' ('to draw'), reflecting the origins of design in divisions of labour for architecture, high volume manufacturing, and remote low volume production. Design became conceived as conceptualisation, with the term 'crafts' progressively applied to millennia old designer-making practices with neither intermediary representations nor separation of conception from realisation. Concerns about design purpose or usage are essentially embellishments without existence beyond the designed artefact. As with human figures around an architectural model, human concerns may be little more than scenic features [15]. Design solely focused on artefacts or usage cannot be human-centred.

However, with no focus on any artefact, there is no design in its craft or conceptualisation senses. Designing as connecting can only extend, and cannot replace, artefact-centred paradigms, adding a parallel focus on design purpose, and a further focus on connecting people and things. Worth maps support both additional foci through human elements (outcomes, UXs) and the connecting associations between these and design elements. Additional worth-centred approaches, notably Worth Boards, add credibility [6] to short MECE labels. Worth board construction further supports identification of innovative value creation and extension, avoiding futile support for existing value in new ways that are no more worthwhile than existing ones. Such worth-centred practices can better home in on design purposes with the most promise, which is hard to achieve through artefact-centred invention.

\subsection{Win 2: Guiding Creativity and Critique}

Worth mapping can no more create design elements than it can human elements. The latter pre-exist in the world, manifested through people, places and things. Design elements either already exist (as inspirations) or must be invented creatively through ideation and technical invention. It would be a mistake to expect worth mapping per se to create design ideas, although it did re-surface some in a context where ideas on possible desirable materials (both hardware and software, e.g., physics engine) and features could be related to both desired value and unwanted costs. Consequently, we avoided the potential trap of justifying and selecting design options on the bases of intrinsic criteria.

Worth maps and UEFs provide better support for connecting and critique than for creation, i.e., they do not support receptiveness [6] to design means or ends. The former are well supported by a wide range of ideation approaches. The latter can be supported via field research (as above) or through innovations for HCI such as the use of sentence completion (e.g., [8]). Worth maps and UEFs support critique through testing out proposed associations between MECEs and within UEF's internal UX dynamics. Once the value of design elements is restrained to the extrinsic, a basis is needed to associate features and qualities to extrinsic value, which both worth maps and UEFs offer. During worth mapping, several initial and proposed features and materials were sidelined until we could plausibly associate them with a design purpose. Overall, worth mapping could make use of a range of separate disciplinary design inputs, both as existing resources (data, analyses, sketches), and as dynamic contributions to discussions. Early on, the project lead (third author) noted how discussions were well balanced, with more frequent confident input from technical roles.

\subsection{Win 3: The Living Document}

Although worth mapping was not completed, the living document that resulted has since been used in a series of further design sessions and is currently still being used as a valuable resource, even in the absence of the facilitator and inventor. The document was primarily maintained as a research tool, providing the process data for this paper, but it has proved to be an effective resource for subsequent design activities. It has also proved to be straightforward to go back to the worth maps document and revise the organising phase worth map, simplifying it as a result of UEFs reducing consequence MECEs to UXs only (Figure 4). Despite a much larger population of design elements, MECs in Figure 4 are much simpler than in Figure 1. Note that associations (red arrows) into the Reliving (Shared) Memories UX differ from those expressed in Figure 3, which is related to design elements and outcomes for the editing phase of Family Archive use.

\subsection{Issue 1: Effective Independent Use}

The mapping facilitator invented worth maps, and could fix them as we went along. Without such an advocate, worth mapping could have failed in the first few sessions. However, the inventor has been minimally involved in recent multiple uses of worth maps in Finland [8]. There have been mixed results here, but even so, worth mapping has shown to be possible and valuable without its inventor present. One use resulted in a video conference presentation of results to all the national offices of an on-line leisure company [8]. Here internal champions for worth mapping could maintain momentum, and have passed on the resulting insights to product owners for implementation. Other uses have been less successful, generally reflecting limited engagement by industrial users and/or limited grounding of human elements due to resource constraints for user research. The challenge here is to simplify the terminology, the process, and the recording of the process, all things that need to evolve through further use. Even in the most successful Finnish use, distinctions between design elements were confusing. Most mapping participants were from business rather than $\mathrm{R} \& \mathrm{D}$ roles, and thus for them, it would have been better to just have product attributes as the sole design MECE type, leaving subsequent $R \& D$ refinements to separate off materials and qualities. Worth mapping thus needs to be sensitive to its participants. Separate consideration of materials, features and qualities was very valuable in the context reported here, since 
Evolving and Augmenting Worth Mapping for Family Archives

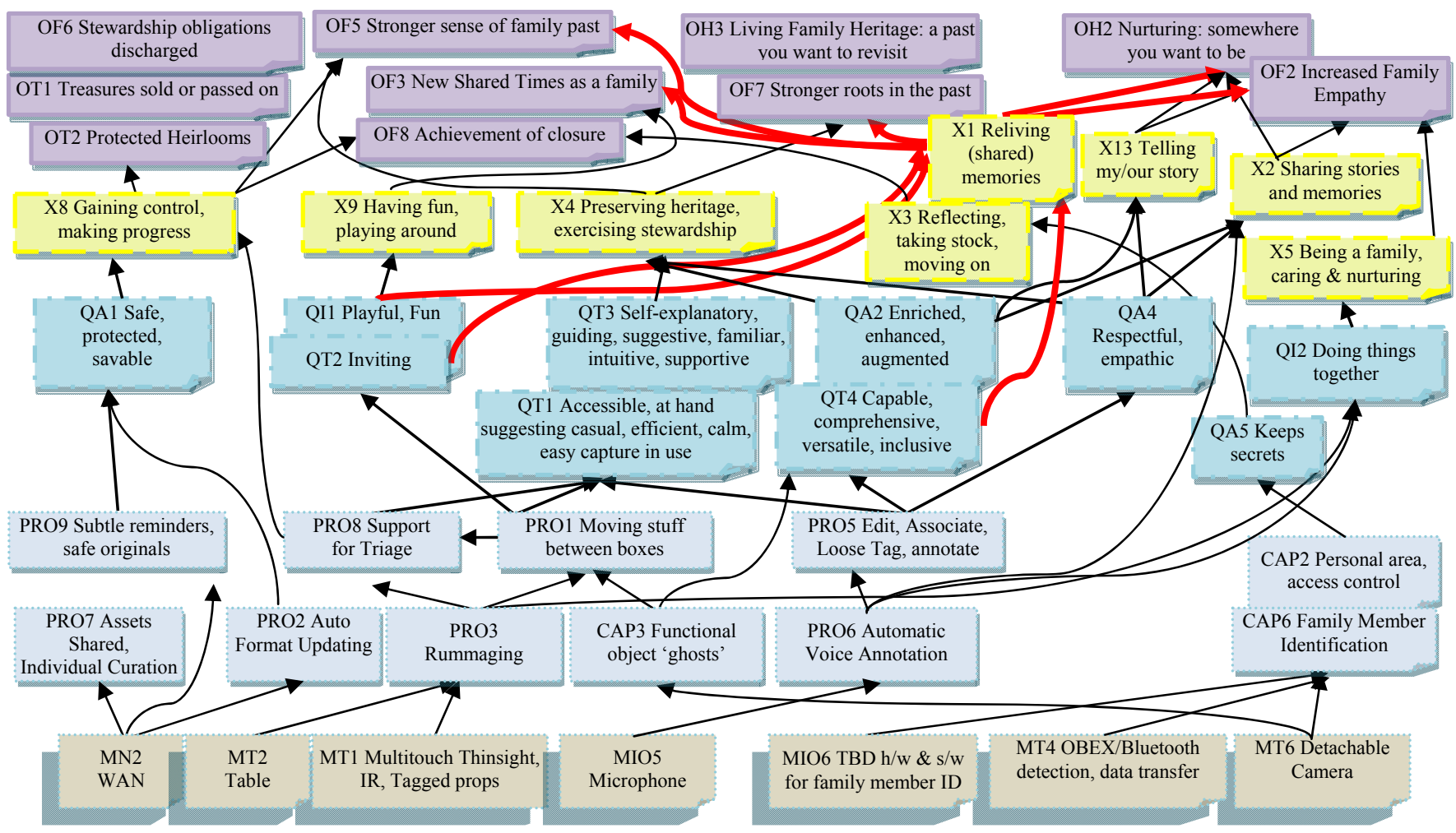

Figure 4: Organising Phase Worth Map, Simplified Through Use of UX MECEs

there were roles who effectively owned each, e.g, materials by hardware engineers, features by software engineers, and qualities by interaction designers, noting that a role's competence can span all three design categories (especially interaction designers).

\subsection{Issue 2: Tool Support}

Use of an inappropriate drawing tool, with MECE lists in a separate living document, led to major inefficiencies. Subsequently, Finnish collaborators have used Visio, which has proved to be more appropriate [8]. Firstly, Visio can link diagrams to a database, and thus MECE names can be systematically updated with no need for manual diagram revision. Secondly, placing all MECEs except UXs in a base layer, and then adding a drawing layer for each UX and its associations, is an alternative to modularisation into separate worth maps for multiple feature groups. The use of layering greatly can greatly reduce the confusing crowd of associations in Figure 4, which remains hard to read and understand, despite the great simplification that resulted from use of UEFs. The use of Visio or a similar dynamic drawing package will be further explored for both worth maps and UEFs. Discount approaches are also being explored, e.g., the use of Word tables for UEFs [7], with one table able to replace a 1000 word scenario. As perhaps a dozen abstract scenarios can be overlaid on one UEF (perhaps by using Visio layers), scores of pages of scenario descriptions may be reduced to one UEF's layered overlays. Worth-centred approaches may thus be viable in fast paced commercial environments. Looking further into the future, large multi-touch multi-user tabletop computers may prove to be a very effective collaborative worth mapping environment, removing the need for house keeping after a mapping session.

\subsection{Issue 3: No Design!}

Design, in the old-fashioned sense of sketching, critiquing, refining and selecting design means paused during the mapping collaboration, frustrating the interaction designer. This was in part due to the constant revisions to MECEs and ultimately worth map structure between sessions, resulting in uncertainty as to whether we had completed any worth map. It was also due to the relatively low frequency of worth mapping sessions, and associated inconsistent attendance. Both were understandable given the speculative nature of the collaboration, but at the same time the lack of follow through sketching and critiques was also an issue of resourcing and scheduling. Where worth mapping can complete (more likely with UX elements and UEFs) and project teams have confidence in interim maps, then time should be allocated to established interaction design practices that refine design elements. Worth mapping could also be put on hold during remedial or extension field work, or during engineering activities required to establish the feasibility of features, or of hardware and software materials. Even so, designing as connecting extends our concept of design to span design means, ends, beneficiaries, evaluations and all the connections between these four categories of design choices [6]. Strictly, 'no design' here means no refinement of design means. Additional design features were added and refined, but only as labeled boxes in worth maps. However, many design ends were identified as outcomes, and both grounded in beneficaries' archiving practices and also related to potential evaluation practices. From the wider perspective of designing as connecting, there was extensive designing across its four choice classes (means, ends, beneficiaries, evaluations: [6]).

\section{CONCLUSIONS AND ADVICE}

Worth mapping is an approach, not a method. It promises no guarantees and depends absolutely on the knowledge and commitment of whoever applies it. It is one approach within a worth-centred development (WCD) framework [6], and it must be complemented with other approaches (e.g., UEFs, Worth Boards) to fully instantiate the six meta-principles that guide WCD [6]. For example, even in the most successful Finnish applications, valuable outcomes were only considered from the user's perspective. Value for the service provider was not in focus, and thus worth mapping was not perceived to provide good support for business strategies [8]. Understanding and instantiating the meta-principle of inclusiveness [6] here 
communicates the need to consider worth from multiple stakeholder perspectives.

The use of worth maps in a multidisciplinary research project team exposed limitations in initial 'post-W/AM' worth maps. As a result, the only common MECE type between current worth maps and their HVM origins is now qualities/defects. A new HCI 'post-task' representation, the UEF, was also invented to overcome major problems with HVM MECEs. This now provides foundations for systematic exploration of UX dynamics, with or without worth maps. Lastly, a first 'discount' instance of a Worth Boards was created, grounded in extensive field data. As a result of this and other recent worth mapping collaborations, the following practices are recommended to project teams who want to use worth maps to support designing as connecting:

1. Combine records with tutorial materials. Create and maintain a living document during the mapping process, with tutorial material on the philosophy underpinning designing as connecting, and the knowledge and concepts associated with MECs and MECE types. It is vital to develop early understanding of MECEs, and buy-in on MECs as a basis for designing as connecting. Failing to do so will most likely result in rejecting worth mapping for the wrong reasons.

2. Modularise. Worth maps get big quickly (even with UEFs). Use practices (e.g., feature subtyping/groups) and/or tool features (e.g., Visio layers) to modularise worth maps.

3. Don't map unsurveyed terrain. Combine worth sketches and maps with other HCI/WCD approaches, especially to ensure receptiveness and credibility [6] in the identification of potential worthwhile and adverse outcomes. Sentence completion was used in [8] and field research in the project reported here. Prior identification of design and/or human elements is essential for effective worth mapping. Also, use UEFs and worth boards to improve expressivity [6].

4. Appropriate all design resources. For example, agree on the MECE types that a project team will use for design elements. Product features may be enough, and materials may not be useful in some projects contexts. Also, don't let a bloated living document kill your project. Keep it lean.

5. Maximise participation. Serving cold worth maps to strangers causes indigestion. Involve as many stakeholders as possible in at least some MECE selection, sketching or mapping sessions. Watching a map being constructed is far easy to follow than trying to read one 'made earlier'. When presenting completed worth maps, use modularisation and tutorial materials as much as possible.

The collaboration thus produced much mutual benefit. To achieve more detailed contributions by refining specific design features and evaluation criteria, future worth mapping needs to proceed more rapidly in conjunction with concurrent design exploration and sketching. Given that the collaboration found and fixed some major problems with worth maps, future use can be more confident, and hopefully worthwhile too, by following the above advice, and better still, generating new advice for others too.

\section{REFERENCES}

1. Aschmoneit, P. and Heitmann, M. 2003. Consumers' cognition towards communities: Customer-centred community design using the means-end chain perspective. Proc. 36th Hawaii Int. Conf. on System Sciences, IEEE.

2. Brown B., Taylor, A., Izadi, S., Sellen, A. and Kay, J. 2007. Locating Family Values: A Field Trial of the Whereabouts Clock. Proc. UbiComp 2007. Springer. 35471.

3. Cockton, G., 2006, Designing worth is worth designing. Proceedings ofNordiCHI 2006, ACM, 165-174.

4. Cockton, G. 2008. Putting value into e-valu-ation. Maturing usability. eds. E. Law, E. Hvannberg and G. Cockton. Springer, 287-317.

5. Cockton, G. 2008. Designing Worth - Connecting Preferred Means to Desired Ends. Interactions. 15(4). 5457.

6. Cockton, G. 2009. Getting There: Six Meta-Princnples and Interaction Design. Proc. CHI 2009, ACM, 2223-2232.

7. Cockton, G. 2009. When and Why Feelings and Impressions Matter in Interaction Design (Invited Keynote). Kansei 2009: Interfejs Użytkownika - Kansei w praktyce, CD Rom Proceedings, Warszawa, Poland.

8. Cockton, G. Kujala, S., Nurkka, P. and Hölttä, T. 2009. Supporting Worth Mapping with Sentence Completion. to appear in Proceedings of INTERACT 2009, Springer.

9. Harper, R. Rodden, T. Rogers, Y., Sellen, A. 2008. Being Human: HCI in 2020. last accessed 5/6/09 at research.microsoft.com/en-us/um/cambridge/projects /hci2020/default.html

10. Ihde, D. 1975. A Phenomenology of Man-Machine Relations, Work, Technology and Education, eds. W. Feinberg and H. Rosemount Jr., University of Illinois Press, 186-203.

11. Kirk, D., Sellen, A., Harper, R. and Wood, K. 2007 Understanding videowork. Conference on Human Factors in Computing Systems. Proceedings of. CHI 2007 , 61-70.

12. Kirk, D., Sellen, A., Rother, C., and Wood, K. 2006. Understanding "photowork". Proc CHI 2006, ACM, 761770 .

13. Marzano, S. 1998. Creating Value By Design: Thoughts And Facts V\&K Publishing.

14. Rokeach, M. 1973. The Nature of Human Values. Free Press.

15. Sharrock, W. and Anderson, B. 1994. The User as a Scenic Feature of the Design Space, Design Studies, 15(1), 5-18.

16. Wilson, A.D., Izadi, S., Hilliges, O., Garcia-Mendoza, A., and Kirk, D. 2008. Bringing physics to the surface. in Proceedings of UIST'08. ACM, 67-76. 